\title{
Use of Blockchain Technology in Agribusiness: Transparency and Monitoring in Agricultural Trade
}

\author{
Papa Semou Faye \\ $\mathrm{PhD}$ student in logistics and supply chain management \\ Wuhan University of Technology (WHUT) \\ Wuhan, China \\ e-mail: fpapesemou@yahoo.fr
}

\begin{abstract}
This paper is exploring the possibilities to use the blockchain technology in agribusiness. Nowadays, the digital transition has resolutely conquered agriculture. Blockchain technology continues to show that it can turn many markets and economies, and agriculture could be one of them. The production growth to meet the demand, lead us to a radical change in agribusiness. Technology can be an important pillar for this transformation. Blockchain management is based on transparency, security and authenticity, could we apply those principles to the new agricultural industry? This study shows that Agricultural sector has a great need for information that support traceability. Each one of the actors concerned must turn to the other part of the value chain to better understand possible external factors and disruptive emerging technologies.
\end{abstract}

Keywords-blockchain; agriculture; transparency; security; traceability

\section{INTRODUCTION}

In 2016, the power of Bitcoins increased drastically all over the world. two third of those Bitcoins were produces in China with blockchain technology. The block chain is an innovative and revolutionary tool that, according to experts, is about to revolutionize our lives. The transformation would be equivalent to the apparition of the press and Internet for the previous generations

The blockchain is just an accounting entry of digital operations, shared among multiple stakeholders. It can only be updated by consensus among most participants in the system. Once entered, the information can never be overwritten. Thus, the bitcoin blockchain contains a certain and verifiable record of each bitcoin transaction ever performed.

Since its creation, it was considered as a precursor of depth change for the organization of worldwide transactions. It seduces governments, large companies, entrepreneurs and investment funds.

Today, many people think that blockchain was created for the currency only. But it can be applied to other areas by implementing a decentralized functioning organization. It is in this context that we opted for the possibilities of using the blockchain in agribusiness and to do this, we organized this article as follow.

We will explain the fundamentals of the blockchain. Then try to see how the Exchange (trading) will be made by highlighting security and transparency, which are essential in the blockchain. Finally, find what are the limits or inconvenience resulting from blockchain adoption in agriculture.

\section{THEORETICAL BACKGROUND}

\section{A. Block Chain Management}

In year 2008, an individual or group writing under the name of Satoshi Nakamoto published a paper entitled "Bitcoin: A Peer-To-Peer Electronic Cash System" [1]. This paper described a peer-to-peer version of the electronic cash that would allow online payments to be sent directly from one party to another without going through a financial institution. Bitcoin was the first realization of this concept.

This system allows people to know everything that happens in the digital world, and with certainty. Today, every interaction you have online relies on a trusted central authority. No matter what you do online, you trust someone to tell you the truth - be it your bank when it gives you your account statement, your email service email when it tells you that your message has been sent, or your antivirus software when it informs you that everything is under control.

There is always the risk that one of the suppliers of information is lying, or is simply mistaken. How do you know when you pay with your credit card online, giving your information, that the merchant site is really secure, that it does not store your banking information and that they will not be hijacked afterwards? You trust him to spend the money needed to secure his site. Which is a bit risky. That's why Internet security is such a big issue; We trust sources that can be hacked, manipulated or compromised. We trust them more and more and we allow it to manage our most personal information and our daily lives.

The block chain could remedy all these defects. This technology allows us to create a real record of events already realized or being realized in the digital world without compromising privacy. You can record the fact that the event took place, and even that it took place correctly, without exposing confidential details about the subject or the stakeholders involved.

This confidentiality explains why there are bitcoin transactions on the black market; Despite the public nature of accounting, users themselves can remain completely 
anonymous. This is one of the main criticism of the blockchain.

\section{B. Agribusiness Supply Chain}

The chains of food value are intended to increase its competitive advantage by collaborating on a project bringing together producers, processors, marketing specialists, food service companies, retailers and support groups, such as shippers, research groups and suppliers. A value chain is a strategic partnership between interdependent firms that maintain collaborative relationships to gradually add value to end consumers, which translates into a collective competitive advantage.

A value chain is characterized by a collaborative, marketoriented: different commercial companies work together to develop and bring to market products and services effectively and efficiently. They allow companies to meet the needs of the market by the production, processing and marketing in line with consumer demand.

\section{BLOCK CHAIN TECHNOLOGY IN AGRIBUSINESS}

\section{A. How Blockchain Works}

The block chain is a storage technology and transparent information transmission, secure, and operating without central control body. By extension, it is a public database, distributed - shared by different users, without intermediaries - reliable and inviolable. ${ }^{[7]}$ Thus, it can be likened to a public ledger account, anonymous and unfalsifiable.

The block chain could eventually replace all the "trusted third party" centralized (banks, notaries, land registers, etc.) by a decentralized computer system. The blockchain technology has features that are already used like the auditable $\log$ of updates, check-pointed transactions or distributed communications.

The block chain performs these functions without central authority. Since it is not managed by a company no one take the decisions and there is no way to remove the data from the blockchain. It is a tool agnostic and was made possible by advances in cryptography, Internet performance and computing power. I think it has revolutionary implications for the world in general and the agricultural and food industry.

\section{B. How to Secure an Exchange in Agribusiness?}

Throughout the transformation chain of raw, exchanges are established between actor two by two: between the farmer and his cooperative, between the cooperative and the transformer, or between the transformer and the dealer. As they are not all present at the time of each Exchange, none of the actors has access to all of the transactions.

To get an accurate picture of the path of a food, therefore a register where all these transactions will be recorded. Anyone can come and consult the register when he wants: it's the notion of transparency. ${ }^{[3]}$

However, to add a transaction to the register with confidence, there that witnesses are present to avoid any misrepresentation. And to avoid any corruption after registration, each witness must leave a copy of the registry.
Thus, a briber should falsify all records without the knowledge of their owners.

Such a solution is utopian in the material world, because it requires to move a lot of world to each transaction to validate registration and copy it back to its registry. Digital cross this obstacle.

\section{Transparency and Safety Dematerialized}

To make it accessible to all, the register is not physical but digital and hosted on the net. It's a giant database anyone can access with his internet connection and without intermediary. One can consult freely and for free each one of the exchanges, present or past, without having to move.

Successive transactions are recorded in the form of "block transactions" which refer to the block above and which, placed end to end, and form a 'chain', where the concept of block Chain. To avoid any falsification of the data stored in the string registry is copy on different computers (or servers) network hosted and accessible on the net. We're talking about network distributes: different copies exist simultaneously on different servers baptized 'nodes' of the network. The registry would be falsified if more than half of these nodes were corrupt simultaneously. The network, the more security is strong.

Writing on servers is secured using cryptographic techniques. Each actor has a private key, only entitled to sign a deal for him. We can't destroy the private from a signature key, so it cannot be intercepted; a complex technique of 'proof of work' to ensure that the network is not drowned in false transaction requests.

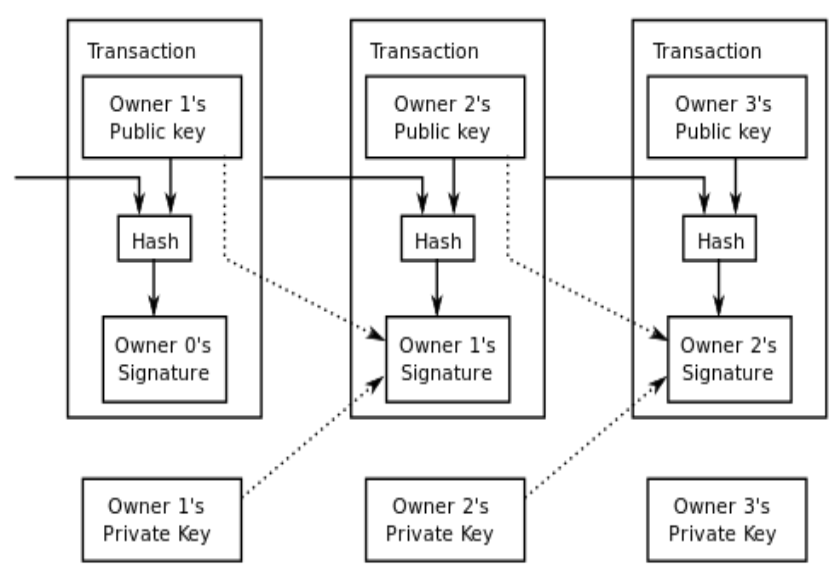

Figure 1. Blockchain transactions (cryptographic techniques )

Finally, before registration, a block is validated by the network. For example, to add a transaction between the cooperative and the transformer, each node verifies that it is the cooperative who signed the transaction and that there is, in its local copy of the registry, a transaction which mentions that farmers have sold their property to the cooperative so it can sell them.

\section{Decentralize the Request}

In networks communication, through an operator to be in relationship with someone a centralized system requires a 
central crossing point. The central authority usually has control over the system and may be the target of attacks.

In a blockchain, there is no privileged interlocutor: the exchanges are matched Peer to Peer. The operators of the chain (nodes) are very numerous and located in many countries on all continents. They share the same computer code, including the open character (code open source) is an additional guarantee of transparency.

A node that would not respect the rules (by changing its computer code) lead to its decision to falsify information.

\section{E. Certification and Traceability in Agriculture}

The current system for agricultural products is an ineffective labeling system that can be easily falsified. Consumers could count on blockchain's transparent book detailing and get all the information's about the food they buy. Thus, this technology concentrates on creating direct relationships while increasing confidence and visibility into the movement of goods.

Blockchain technology could manage the agricultural trade while providing guarantees in the certification procedures.

Since it secures any transaction, blockchain replaces traditional trusted third parties: Bank, notary... Therefore countries, victims of corruption where trusted third parties are not reliable, are working to save the land titles on the blockchain.

In agriculture, the blockchain could manage trade while providing guarantees in procedures for certification of their quality or origin. For instance, the radio frequency identification is a system that allows to store and retrieve data remotely through sensors (tailbacks, the monitoring of books in libraries, banking services, biometric passports, the access to transport). ${ }^{[10]}$ These traceability techniques will help to capture, store and manage all the information of the product. If a product is identified as non-compliant, the market players must demonstrate that they have met stringent quality standards.

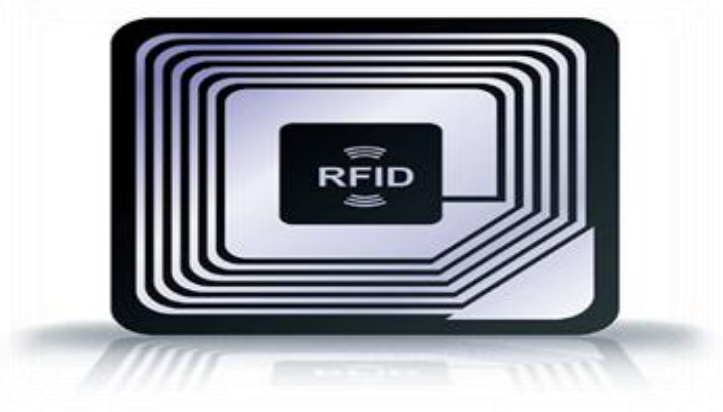

Figure 2. Radio-frequency identification

Finally, the blockchain promises to not only be limited to the simple recording of transactions but also executing computer programs. The latter will allow automatic execution of smart contract defined beforehand (smart contact). For example, to trigger the payment of insurance as soon as these programs identify the conditions are met. The blockchain will therefore simplify the work of all actors in the "Agri-chain" and make transactions more transparent.

\section{CONCLUSION}

Before The aim of this paper was to consider the application of the blockchain into the agricultural industry. We have seen that it will foster more transparency and traceability in agriculture and thus re-establish the confidence of consumers in agricultural products. Nevertheless, all these promises are accompanied by anxiety from different actors related to the dematerialization of documents or the consumption of energy.

However, the technology itself cannot save the planet. It is essential to deeply change the culture and the political will if we want to put in place a successful and transparent agricultural system that ensures a healthy and sustainable food for all.

\section{ACKNOWLEDGMENT}

I would like to show my gratitude to Mr. Song, Wuhan University of Technology for giving me a good guideline for assignment throughout numerous consultations. I would also like to expand my deepest gratitude to all those who have directly and indirectly guided us in writing this paper.

\section{REFERENCES}

[1] Satoshi Nakamoto "Bitcoin: A Peer-To-Peer Electronic Cash System",2008.

[2] W. Dai, "b-money," http://www.weidai.com/bmoney.txt, 1998.

[3] Reid, F., Harrigan, M. An analysis of anonymity in the bitcoin system. In: Security and Privacy in Social Networks. 2013.

[4] "Chain | Enterprise Blockchain Infrastructure." Chain Enterprise Blockchain Infrastructure. N.p., n.d. Web. 03 May 2016.

[5] Miers, I., Garman, C., Green, M., Rubin, A.D. Zerocoin: Anonymous distributed e-cash from bitcoin. In: Proceedings of the IEEE Symposium on Security and Privacy. 2013.

[6] C. Decker, R. Wattenhofer, Information propagation in the bitcoin network, in: 13th IEEE Conference on Peer-to-Peer Computing, 2013, pp. 1-10.

[7] D. Tapscott, A. Tapscott Blockchain Revolution: How the Technology Behind Bitcoin is Changing Money, Business, and the World (2016) (Portfolio)

[8] L. Qiu, H. Rui, A.B. Whinston The impact of social network structures on prediction market accuracy in the presence of insider information J. Manag. Inf. Syst., 31 (1) (2014), pp. 145172

[9] H I. Eyal, E.G. Sirer Majority is not enough: bitcoin mining is vulnerable International Conference on Financial Cryptography and Data Security, Springer, Berlin Heidelberg (2014), pp. 436454

[10] S. Chai, M. Kim, H.R. Rao Firms' information security investment decisions: stock market evidence of investors' behavior Decis. Support. Syst., 50 (4) (2011), pp. 651-661

[11] C. Tucker Identifying formal and informal influence in technology adoption with network externalities Manag. Sci., 54 (12) (2008), pp. 2024-2038. 